\title{
Oscillatory Rayleigh-Bénard Convection in elasto-viscoplastic gels
}

\author{
Christel Métivier \\ Laboratoire d'Energétique et de Mécanique Théorique et Appliquée (Université de \\ Lorraine, CNRS), France \\ Frédéric Brochard \\ Institut Jean Lamour (Université de Lorraine, CNRS), France \\ Mohamed Darbouli, Albert Magnin \\ Univ. Grenoble Alpes, CNRS, Grenoble INP, LRP, F-38000 Grenoble, France
}

\begin{abstract}
Rayleigh-Bénard convection (RBC) is studied in an elasto-viscoplastic (EVP) gel, the widely used and studied Carbopol gel. The transition from conductive to convective regime is determined by the Schmidt-Milverton principle which leads to a critical value of the inverse of the Yield number $1 / Y_{c}$. However, an oscillatory motion in the gel is observed below $1 / Y_{c} \approx 60$ when smooth and untreated walls are used. Particles within the gel describe periodical loops close to their initial positions for $16.5<1 / Y<25$. For larger values of $1 / Y \approx 50<1 / Y_{c}$, loops are still obtained but particles move away progressively from their initial position. The oscillations take the form of periodic traveling waves $(\mathrm{TW})$. We demonstrate that elastic effects play the major role in the occurence of oscillations.
\end{abstract}

\section{Introduction}

In a review of Rayleigh-Bénard convection in viscoelastic fluids in the 1990's, Larson [1] wrote "viscoelastic effects are unimportant in the linear stability of Rayleigh-Bénard flow, except perhaps under extraordinary conditions". He would be certainly right because linear stability analyses [2, 3, 4] of a viscoelastic fluid layer heated from below show that in most cases a stationary convection is obtained above the critical Rayleigh number $R a_{c}$ which 
value is similar to the Newtonian case. However, under some specific conditions, an overstable mode can develop, leading to an oscillatory convection below $R a_{c}$ for the particular case of viscoelastic fluids. For a Maxwell fluid, it is shown that the occurence of such a mode implies high Prandtl number $\mathrm{Pr}$ - ratio between kinematic velocity and thermal diffusivity - and a Deborah number $D e$ - ratio between the relaxation time and the thermal diffusivity time of the fluid - such as $D e \geq 0.03$. Larson explained that the fluid must have a long relaxation time, the fluid layer height $h$ must be of several $\mathrm{cm}$ and the temperature difference between the two horizontal plates should be around $\Delta T=50 \mathrm{~K}$. In the unlikely case where oscillations occured, their frequency would be $\omega_{c}<10^{-3} \mathrm{~Hz}$. To our knowledge, only one experimental study reported by Kolodner [5] highlights an oscillatory convection in a viscoelastic fluid, a DNA suspension. However, it is not sure that the oscillatory convection is only due to elastic effects. In his study, Kolodner claims that oscillations could also be due to the migration of the DNA polymer in the solution, i.e. that the Soret effect could play an important role. It renewed the interest for the oscillatory convection in (binary) viscoelastic fluids till now.

Elasto-viscoplastic gels can also present significant elastic effects. In addition to viscoelastic effects, they also present an important feature, namely the yield stress $\tau_{y}$ below which the gel has a solid-like behavior and above which it flows with a shear-thinning behavior usually described by the HerschelBulkley law $\tau=\tau_{y}+K \dot{\gamma}^{n}$, where $\tau$ is the stress, $\dot{\gamma}$ the rate of strain, $K$ and $n<1$ are respectively the consistency and the shear-thinning exponent. The yield stress materials, especially concentrated suspensions, promote slip at solid surfaces rather than deformation or shear flow [6, 7]. Slip at walls of microgel suspensions has been the subject of extensive experimental and theoretical studies in rheometric flows $[8,9,10,11]$.

The Rayleigh-Bénard convection in viscoplastic fluids has been studied by several researchers $[12,13,14,15,16,17]$ in the beginning of this century, however theoretical studies never consider elastic yield stress fluids. This is probably because yield stress fluids are usually modeled by inelastic models such as the Bingham and the Herschel-Bulkley ones. [12, 13] show that inelastic viscoplastic fluids heated from below are linearly stable. Furthermore, it was not clear that elasticity had a major effect on the onset of convection regarding the yield stress effect. In previous experimental studies [14, 15, 17], it is shown that the governing parameter is the ratio between buoyancy and yield stress effects, i.e. the inverse of the Yield number $1 / Y=(\rho g \beta \Delta T h) / \tau_{y}$, 
with $\rho$ the fluid density, $g$ the gravitational acceleration, $\beta$ the thermal expansion coefficient. It is also shown that steady convection in Carbopol gels occurs above the threshold value $1 / Y_{c} \approx 80$ for no-slip boundaries and that $1 / Y_{c}$ is smaller for slip boundaries, for instance Darbouli et al. [14] get $1 / Y_{c} \approx 60$ for $\tau_{y}=0.03 \mathrm{~Pa}$. These previous studies focus on the determination of $1 / Y_{c}$ and the characterization of the steady convection. There is no mention to any oscillatory motion below $1 / Y_{c}$. In the present paper, we focus on the subcritical conditions and highlight the occurence of an oscillatory convection in the only case of slip at walls.

\section{Materials and methods}

The elasto-viscoplastic gel is obtained by mixing Carbopol 940 powder, manufactured by BF Goodrich, in distilled water. Carbopol is made from reticulated polyacrylic acid resins. This solution does not present any nonNewtonian feature, it has to be neutralized with $\mathrm{NaOH}$ in order to involve polymer swelling and microgel formation. During the neutralization and during several hours the gel is gently mixed (around $100 \mathrm{rpm}$ ). The gel is then stored at $4{ }^{\circ} \mathrm{C}$ during two days. In the present paper, we study one Carbopol gel (Carbopol 940 at a weight concentration of $0.047 \%$ in distilled water).

The bulk rheological properties of the aqueous gel were measured using a TA Instrument rheometer (AR-G2) with an angular resolution of $10^{-8}$ rad and a torque resolution of $10^{-10} \mathrm{Nm}$. A cone-plane geometry was used. The controlled speed is imposed on the upper tool. All measurements are performed at $293 \mathrm{~K} \pm 1 \mathrm{~K}$. To avoid gel slippage at the interfaces, the coneplane geometry is roughened by sticking sandpaper to the geometry surfaces. The sandpaper has a mean roughness of $125 \mu \mathrm{m}$ and is water resistant. This technique is well known for roughening surfaces and is very effective in ensuring fluid adhesion to the wall. No-slip conditions of the lower plane can also be obtained by chemically treating the surface. Here, the treatment corresponds to add a thin layer of PEI on a PMMA or glass surface as proposed by Métivier et al [18]. In order to verify that treated surfaces lead to no-slip conditions, we performed rheometric tests by keeping the rough cone (sandpaper), and replacing the lower plane by the interfaces used in the Rayleigh-Bénard experiments i.e.. treated PMMA, and treated glass [18]. Figure 1 shows the evolution of the shear stress as a function of the shear rate at steady state, for the gel used and for the different treated surfaces. It can 


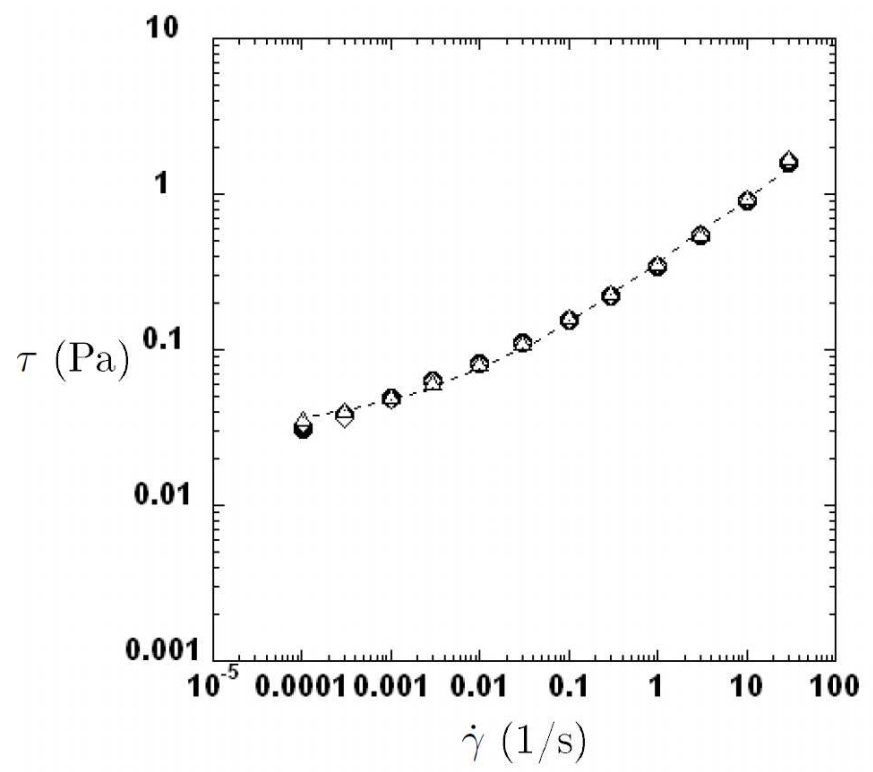

Figure 1: Flow curves of the bulk Carbopol gel for different surfaces (Black circles: sandpaper, White triangles: treated PMMA, White rhombs: treated glass, Dashed line: HerschelBulckley model). Rheological properties of the Carbopol gel $\tau_{y}=0.03 \mathrm{~Pa}$ to $\mathrm{T}=293 \mathrm{~K}$.

be seen that the flow curves are similar for all surfaces used. The flow curve (Figure 1) is typical of a viscoplastic fluid as it follows the Herschel-Bulkley model. It leads to $\tau_{y}=0.03 \mathrm{~Pa}, K=0.26 \mathrm{~Pa} . \mathrm{s}^{n}, n=0.46$. These properties do not make it possible to fully characterize the Carbopol gels. Below the yield stress, the properties of the gel are determined by oscillatory rheometry. Figure 2(a) represents the loss modulus $G^{\prime \prime}$ and elasticity modulus $G^{\prime}$ as a function of the deformation at fixed frequency $f=0.1 \mathrm{~Hz}$. At low strain values, the constant values of $G^{\prime}=0.77 \mathrm{~Pa}$ and $G^{\prime \prime}=0.23 \mathrm{~Pa}$ correspond to the linear viscoelastic domain. Beyond this, the moduli decrease and cross at a characteristic value $\gamma_{c}=0.3$. The stress value $\tau_{c}$ evaluated at $\gamma_{c}$ by $\tau_{c}=G^{\prime} \gamma_{c}\left(G^{\prime}=G^{\prime \prime}\right)$, is a good estimate of the yield stress $\tau_{y}$. In the case of the tested gel, we find $\tau_{c}=0.03 \mathrm{~Pa}$, which verifies well $\tau_{c} \approx \tau_{y}$. The Figure $2(\mathrm{~b})$ displays $G^{\prime}$ and $G^{\prime \prime}$ moduli vs. $\omega$ the oscillatory pulsation at a constant strain $\left(\gamma<\gamma_{c}\right)$. We observe that $G^{\prime}$ is always dominant in the linear domain and there is no intersection with $G^{\prime \prime}$ in the range of tested pulsations, highlighting that Carbopol gel has a dominant elastic behavior in the linear domain. 


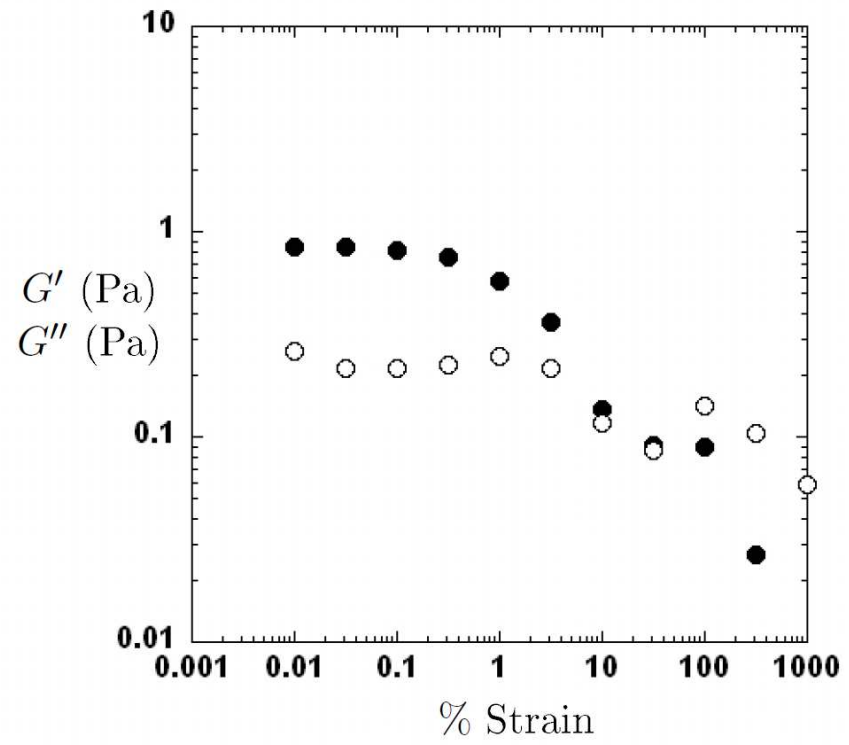

(a)

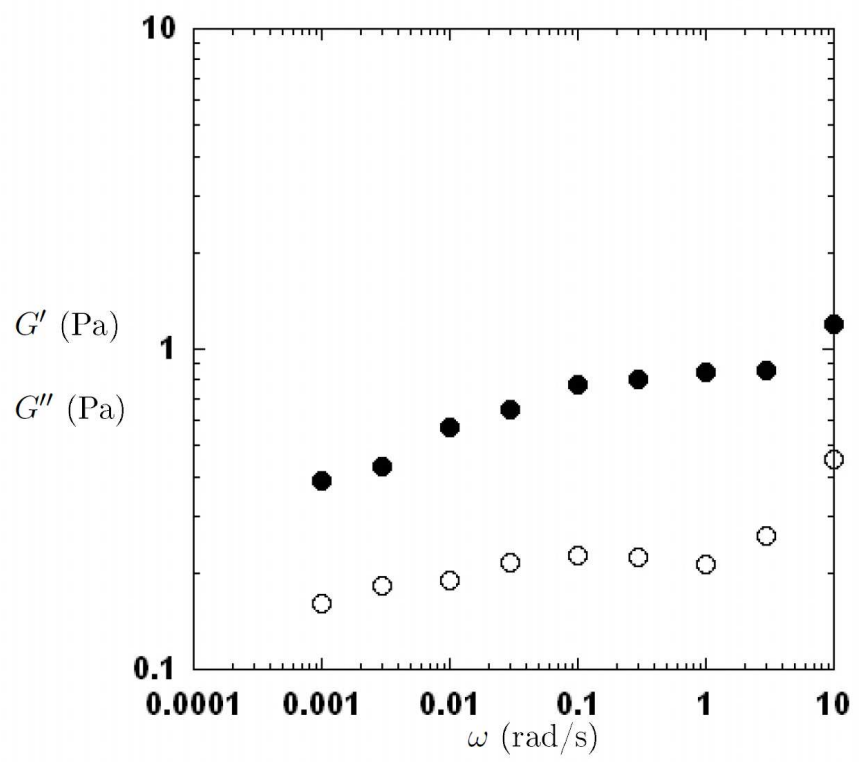

(b)

Figure 2: Oscillatory rheometry of the Carbopol gel (a) Variations of $G^{\prime}$ (Black Circles) and $G^{\prime \prime}$ (White Circles) as a function of deformation at frequency $f=\omega / 2 \pi=0.1 \mathrm{~Hz}$ (b) Pulsation sweep for $\gamma=1 \%$. 
In the series of measurements shown in Fig. 3, the no-slip conditions are maintained at the cone (sandpaper), while the lower surface is successively replaced by the surfaces used in the Rayleigh-Bénard cavity, i.e. the untreated PMMA, glass, and copper surfaces. The results presented in Fig.3 highlight the sliding of a gel $\left(\tau_{y}=0.031 \mathrm{~Pa}\right)$ on these surfaces, through the decrease of $\tau$ values below $\tau_{y}$ for low shear rate values. The modification of the curves and the value of the threshold stress depend on the nature of the surface used. It has been shown that the slip is in a very thin layer close to the solid interface. When the gel is sheared between the rough surfaces, the flow is homogeneous throughout the volume and the gel adheres at the interfaces. The gel is characterized by the yield stress $\tau_{y}$, which is a bulk property. When the gel is sheared between a rough surface and a smooth surface under low stress, the shear is inhomogeneous. The gel adheres to the rough cone but slides on the smooth surface. Slippage occurs from a frictional stress $\tau_{f}$, which is less than the bulk yield stress, i.e. $\tau_{f}<\tau_{y}$. For large shear rate values, and whatever the nature of the interface used, slip becomes negligible with respect to shear rates and the curves coincide with that obtained with rough surfaces. In the presence of slip, Fig. 3 represents an average of shear stress as a function of an apparent shear rate, proportional to the interfacial slip velocity, at low values of stress.

As proposed by several authors $[8,9,19]$, the friction model, in the unidirectional case, is defined by the following equation:

$$
\tau=\tau_{f}+c_{f} \dot{\gamma}^{m} \quad \Leftrightarrow \quad \tau>\tau_{f}
$$

where $c_{f}$ is a coefficient which depends on the nature of the surface, $\dot{\gamma}$ represents the apparent shear rate proportional to the sliding speed $U_{s}$ at the lower interface $\left(\dot{\gamma}=U_{s} / h\right.$ at the outer radius of the cone $R, h$ being the gap), $m$ is a power law parameter. Note that when $\tau_{f}=0$, we find the classical Navier law. In the case of the gel used, we find $\tau_{f}=0.021 \mathrm{~Pa}, c_{f}=1.35$ Pa.s ${ }^{m}$ and $m=0.77$ for the copper surface; $\tau_{f}=0.013 \mathrm{~Pa}, c_{f}=0.65 \mathrm{~Pa} . \mathrm{s}^{m}$ and $m=0.75$ for the glass surface. Values of $m$ are constant for all surfaces, differences in values are obtained for $\tau_{f}$ and $c_{f}$. As expected, the respective values of $\tau_{f}$ and $c_{f}$ follow the same trends, i.e. their maximum value is obtained for the copper surface, their minimum value for the glass surface. This means that the glass surface is slightly more slippery than the copper and PMMA surfaces. Note that a microscopic approach to the sliding of microgel suspensions was proposed in several articles $[7,8,9,10]$ and applied to the Carbopol gel by Ahonguio et al. [20] for instance. 


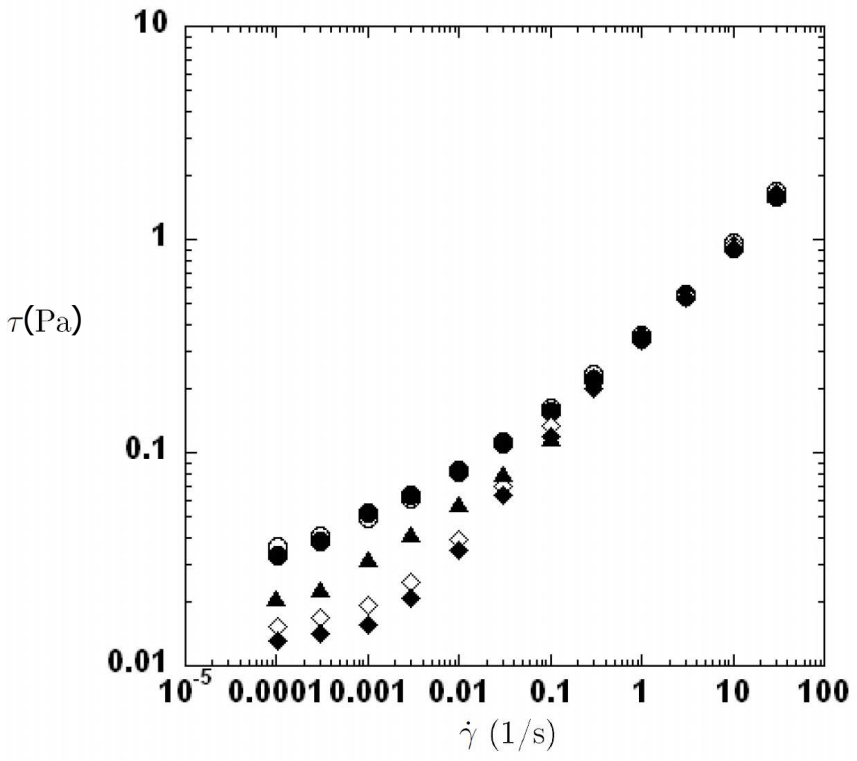

Figure 3: Flow curves for a Carbopol gel $\left(\tau_{y}=0.03 \mathrm{~Pa}\right)$ when different bottom surfaces are used, $\mathrm{T}=293 \mathrm{~K}$. In the case of no slip conditions (Black circles: sandpaper, White circles: treated PMMA). In the case of slip conditions (Black Diamonds: Glass, Black Triangles: Copper, White Diamonds: Rough PMMA), results represent an average of the apparent shear stress vs. an apparent shear rate. 


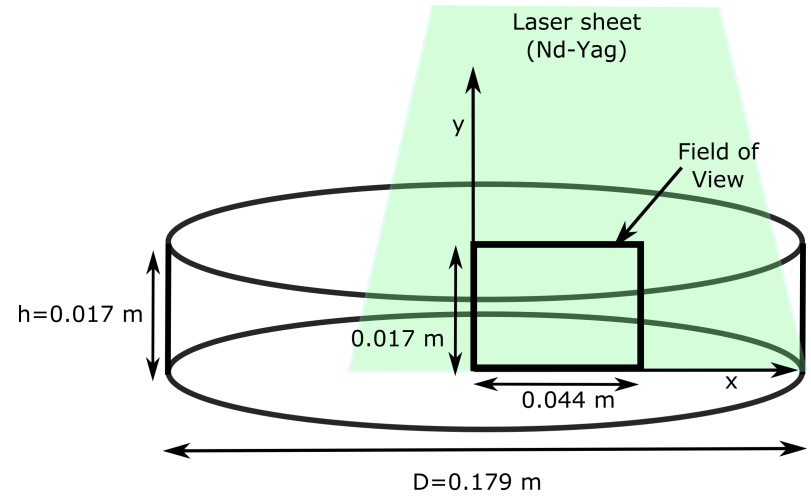

Figure 4: Scheme of the experimental device

As verified by our rheological measurements, the Carbopol gel used in this study is considered as a simple yield stress fluid, in the sense that it does not involve any thixotropic behavior or significant shear-banding [21, 22, 23].

The experimental device, depicted in Fig. 4, is the same as that of our previous studies $[14,17]$, it consists in a cylindrical cavity which aspect ratio is $A=D / h=0.179 / 0.017=10.5$. The fluid layer is heated from below via a heat flux and cooled from above via a temperature controlled water flow, the lateral wall is considered as adiabatic. The lower surface is made of copper, the upper surface is made of glass.

In addition to previously reported experiments, hollow silver spheres supplied by Dantec ${ }^{1}$ (mean radius $r=8 \mu \mathrm{m}$ ) have been mixed in the Carbopol gel in order to probe the flow. They are enlightened from the top by a vertical laser sheet (Nd-Yag, with a wavelength of $1064 \mathrm{~nm}$ and a thickness of $1 \mathrm{~mm})$. It enables us to observe the particles in a vertical plane defined in Fig. 4 by the field of view, it includes the vertical cylinder axis $y$ (center of the cavity) and the horizontal plates along a radial direction $x$.

\section{Results}

A first way to determine the onset of convection is to use the SchmidtMilverton principle [24]. It consists in considering $\Delta T$ vs. $Q_{T}$, the heat

\footnotetext{
${ }^{1}$ We have checked that hollow silver spheres with a density of $1100 \mathrm{~kg} / \mathrm{m}^{3}$ can not be subject to sedimentation in the range of our experiments.
} 


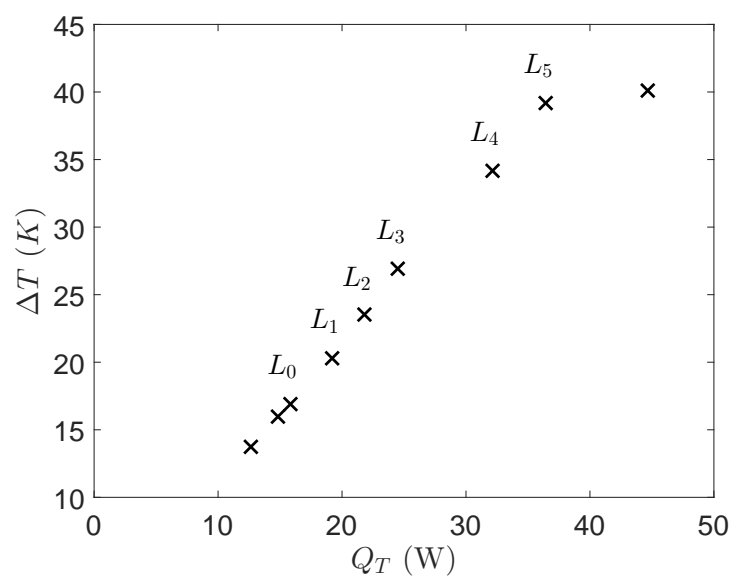

Figure 5: $\Delta T$ vs. $Q_{T}$ for the Carbopol gel $\tau_{y}=0.03 \mathrm{~Pa}$ - slip boundaries. L0: $1 / Y=16.5$, L1: $1 / Y=17, \mathrm{~L} 2: 1 / Y=23, \mathrm{~L} 3: 1 / Y=25, \mathrm{~L} 4: 1 / Y=41, \mathrm{~L} 5: 1 / Y=55$.

flux at the lower plate, as done in Fig. 5 in the case of slip boundaries. The threshold value $\Delta T_{c}$ is characterized by a change in the curve slope corresponding to the transition from conductive to convective regime.

In the conductive regime the total heat flux $Q_{T}$ is proportional to the temperature difference between the horizontal walls $\Delta T$; above $\Delta T_{c}$ the convection occurs and it is characterized by a significant decrease in the curve slope as observed in Fig. 5. This figure displays $\Delta T$ vs. $Q_{T}$ in the case where walls are untreated (slip conditions). The Nusselt number $N u=Q_{T} \times$ $h /\left(S \lambda_{c} \Delta T\right)$, with $S=\pi D^{2} / 4$ and $\lambda_{c}$ the thermal conductivity of the fluid, is also evaluated. For $\Delta T<\Delta T_{c}$, we find that $N u \approx 1$ which should confirm that the regime is conductive. In the case of no-slip boundaries obtained by treating every surfaces in the cavity as explained in Métivier et al. [18], we have not observed any motion within the gel below the threshold temperature difference $\Delta T_{c}$. However, when walls are untreated (slip conditions), the tracking of probe particles in the gel highlights a motion in the gel.

The video data have been processed with the TRACK software developed by Baude \& Desecures [25] from APREX Solutions, a PTV software capable of efficiently analyzing data with low Signal-to-Noise ratio. In our case, the best results have been obtained by combining the detection method based on auto-adaptative thresholding [26] with the predictive tracking based on Bayesian methods [27]. The first technique is a multi-scale thresholding method, which is well suited when the signal-to-noise ratio is unfavorable or 
the lighting is inhomogeneous, as is our conditions. The images are analyzed at 4 different spatial scales (from the pixel to the whole image), allowing to define a different threshold value at each point. The second technique allows to predict the trajectory of an object by smoothing its position, speed and acceleration at each instant using Bayesian probability theory, thus eliminating disturbances due to measurement errors and noise. As a result, several tens of particles are correctly tracked over the entire videos, making it possible to carry out detailed analysis. The TRACK software provides at each time, the position $x, y$ and the velocity vector $v_{x}, v_{y}$ of tracked particles. For sake of clarity, some particles trajectories are selected and displayed in Fig. 6. These results are in very good agreement with trajectories obtained by adding raw recorded images furing the time $t_{t}$ of the experiment as displayed in Fig. 7. We note that some light reflection occurs at the bottom surface (smooth copper plate) which results in a somewhat reduced accuracy of particle tracking around $y=0$. We can observe that trajectories consist in periodic loops with amplitude of displacement of the order of few mm. The tracked particles remain in the laser sheet during at least two periods. The particles displacement increases with $1 / Y$. For small values of $1 / Y(1 / Y<25)$, each particle remains close to its initial position and nearly goes back to its initial position after one period. For larger values of $1 / Y$ and still below the critical value, loops are still obtained but a continuous drift is observed, i.e. particles move away progressively from their initial position. The spatial average velocity magnitude denotes amplitudes of the order of $10^{-5} \mathrm{~m} / \mathrm{s}$ and an oscillation phenomenon as depicted in Fig. 8. Due to the weak motion, we could expect that the material behaves as a solid (elastic solid or elastoplastic solid as described by Coussot [30]). However, the evaluation of the angle $\alpha\left(\vec{e}_{x}, \vec{e}_{y}\right)=C_{x y} / \sqrt{C_{x x} C_{y y}}$ where $C_{i j}$ is the right Cauchy-Green tensor components, leads to very large values of deformation of elements which were initially parallel to $x$ and $y$ directions. The maximal values of $\alpha\left(\vec{e}_{x}, \vec{e}_{y}\right)$ tend to $\pi / 2$, leading to very large values of strain $\gamma$ since $\gamma=\tan \alpha$. It means that the gel is sheared, i.e. fluidized (liquid-like), rather than only subject to small strain (solid-like). This is confirmed by the evaluation of the strain rate tensor components $\dot{\gamma}_{i j}$ : the maximal values of the second invariant $\dot{\gamma}$ is the order of $10^{-2} \mathrm{~s}^{-1}$ for all values of $1 / Y$ while it increases slightly with $1 / Y$.

The oscillations observed in Figs. 6 and 8, can be characterized by a spatial average period $P$ corresponding to the mean time for a particle to 


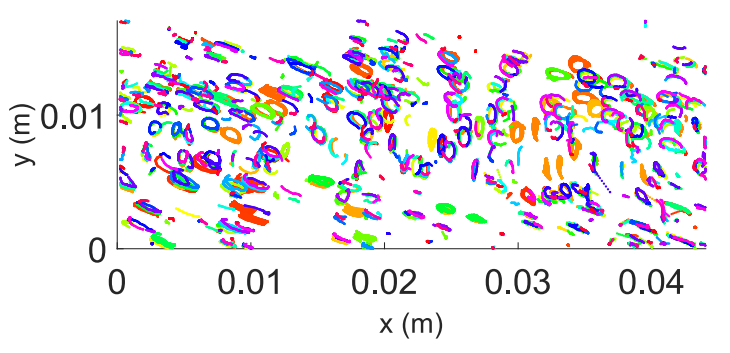

(a) $1 / Y=16.5$

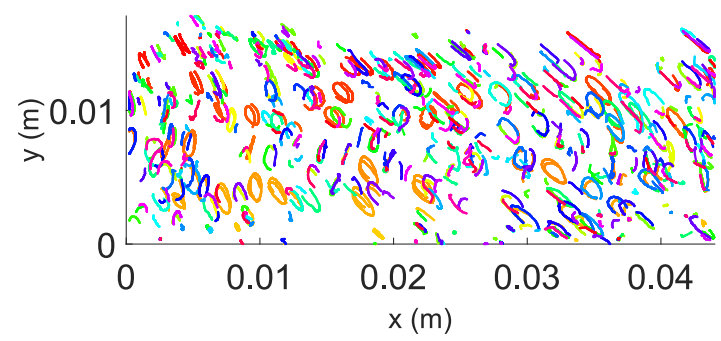

(b) $1 / Y=25$

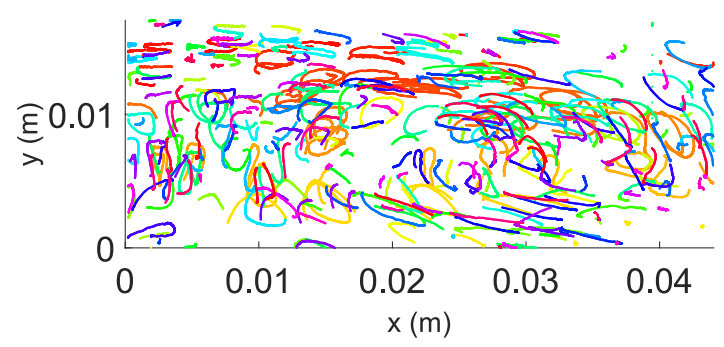

(c) $1 / Y=55$

Figure 6: Trajectories obtained with TRACK in the gel $\left(\tau_{y}=0.03 \mathrm{~Pa}\right)$ for (a) $1 / Y=16.5$, (b) $1 / Y=25$ and (c) $1 / Y=55$. One color corresponds to one tracked particle. For a sake of clarity, all tracked particles trajectories are not represented. 


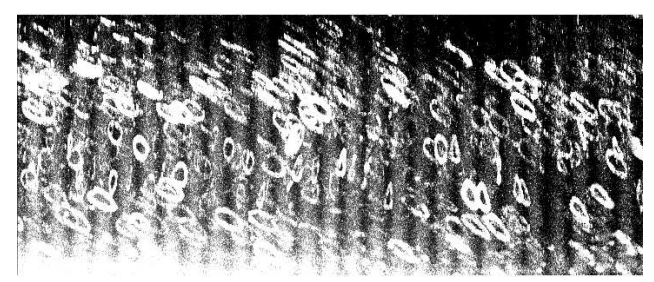

(a) $1 / Y=16.5$

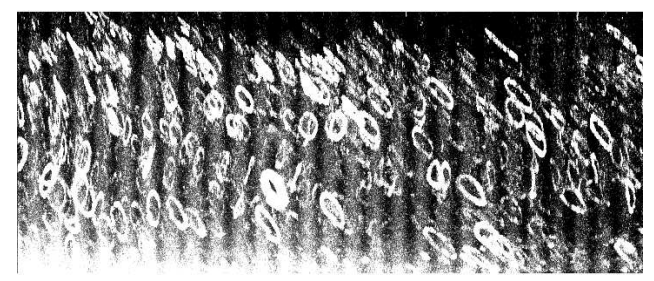

(b) $1 / Y=25$

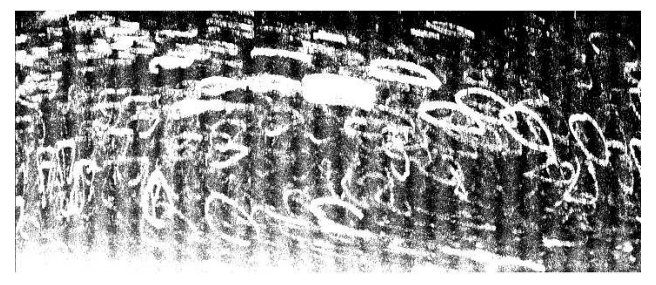

(c) $1 / Y=55$

Figure 7: Trajectories (white) obtained by adding recorded images during the time $t_{t}$ of the experiment for (a) $1 / Y=16.5, t_{t}=360 \mathrm{~s}$, (b) $1 / Y=25, t_{t}=440 \mathrm{~s}$ and (c) $1 / Y=55$, $t_{t}=520 \mathrm{~s}$. 


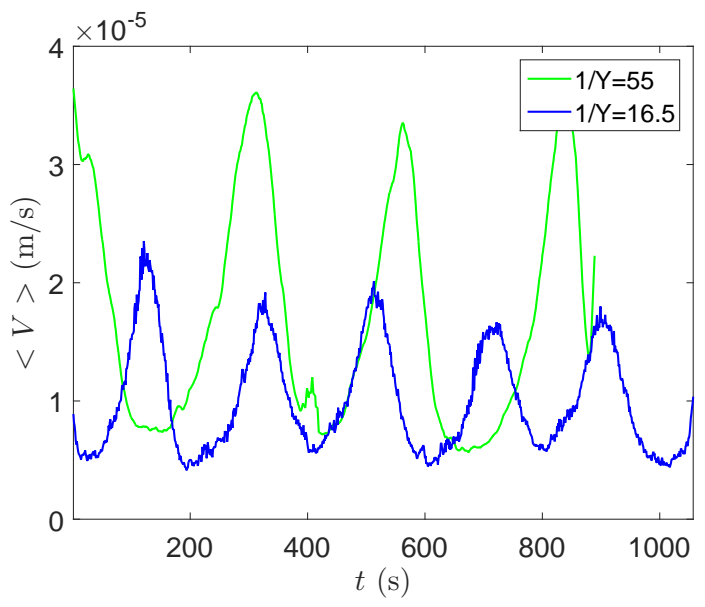

Figure 8: Spatial average over the FOV of the velocity magnitude $\langle V\rangle$ vs. time for $1 / Y=16.5$ and $1 / Y=55$

describe one loop. As depicted in Fig. 9, $P$ increases with the increase in $1 / Y$, with a law $P \propto 5 / Y$, meaning that one particle takes more time to describe a loop when the buoyancy is increased. This feature is associated to an increase in the distance covered by a mean particle, i.e. around 4-5 millimeters per period for $1 / Y<25$ to the order of a centimeter per period for $1 / Y=55$. It leads to a space-time averaged particle velocity around $10^{-5}$ $\mathrm{m} / \mathrm{s}$ for $1 / Y<25$ and around $2.10^{-5} \mathrm{~m} / \mathrm{s}$ for $1 / Y=55$.

It is worth noting that the oscillatory regime is characterized by long oscillations period $P$ and weak velocity amplitude. This motion occurs in a state which would be expected for conduction, i.e. a motionless state. The time-averaged Nusselt number $N u$ is consistent with the value that one would expect for steady-state conduction since one obtains $N u \simeq 1$ and weak fluctuations in $N u$ for long times. This means that the oscillatory motion in the gel has an infinitesimal amplitude inducing only a very small variation in heat transfer. A Fourier transform of the time variations of $N u$ leads to a frequency of the order of $10^{-3} \mathrm{~Hz}$. This is in agreement with frequencies obtained from the particles velocity fluctuations $\omega=1 / P$.

Considering the structure of the flow via the velocity vectors at different time (Fig. 10), we observe the onset of counter-rotative large convective cells. These cells are traveling with time as underlined by the motion of their boundaries (blue or red dashed lines) in Fig. 10. The traveling waves (TW) are also highlighted in Fig. 11 which displays space time diagrams of 


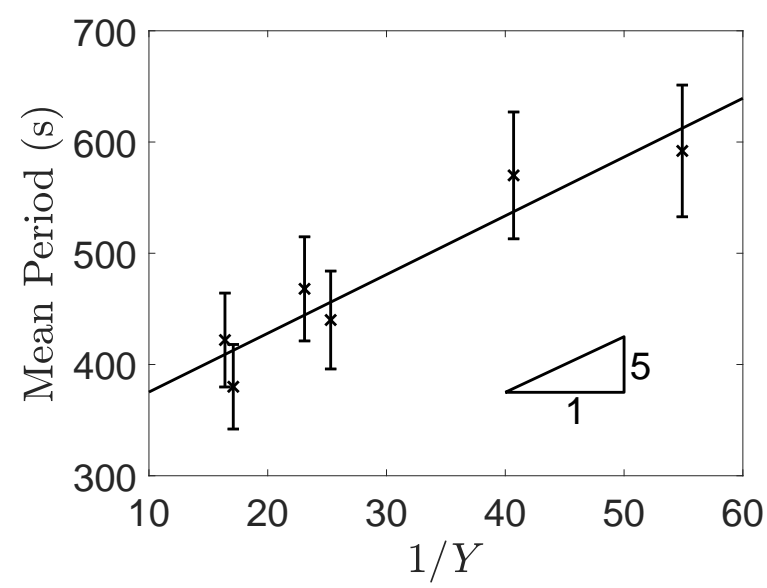

Figure 9: Spatial average period $P$ as a function of $1 / Y$
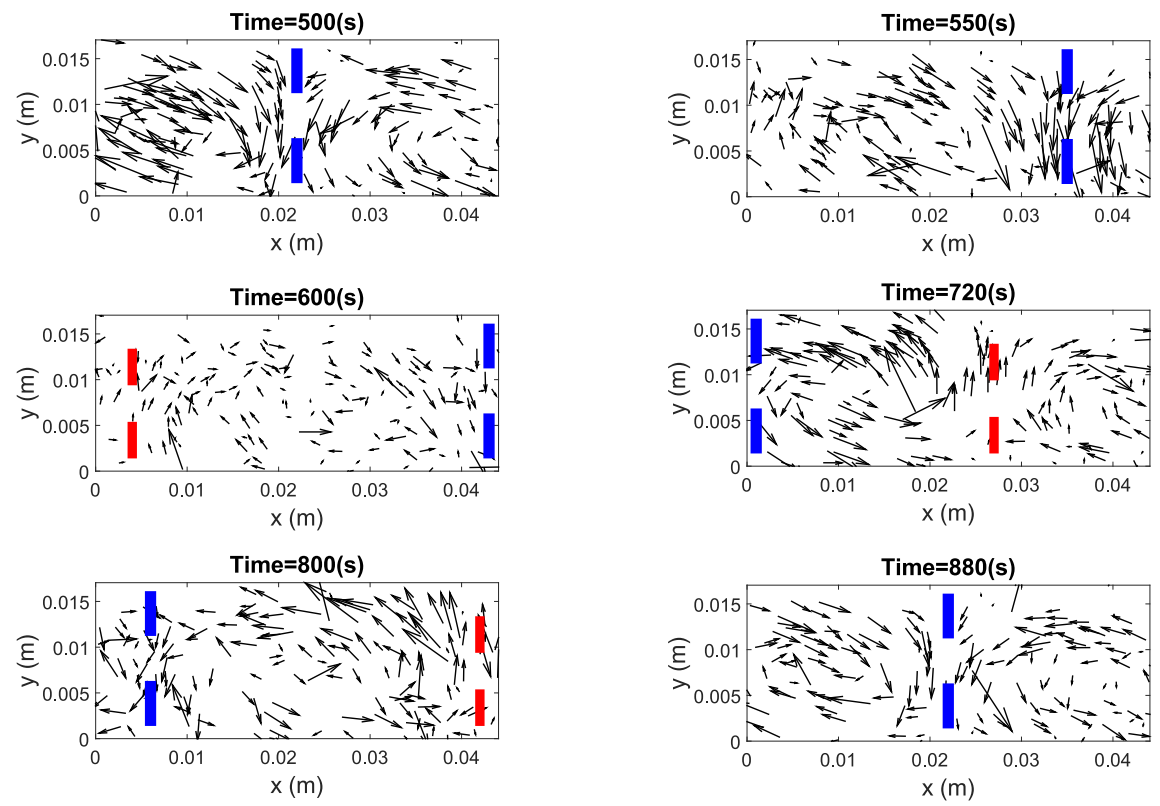

Figure 10: Velocity vectors during a period for $1 / Y=16.5$ - Dashed lines: approximate boundaries between two counter-rotative cells, Blue for the lower flow and Red for the upper flow. Oscillations take the form of traveling waves (TW) moving from left to right in this case $\left(v_{p}>0\right)$ 


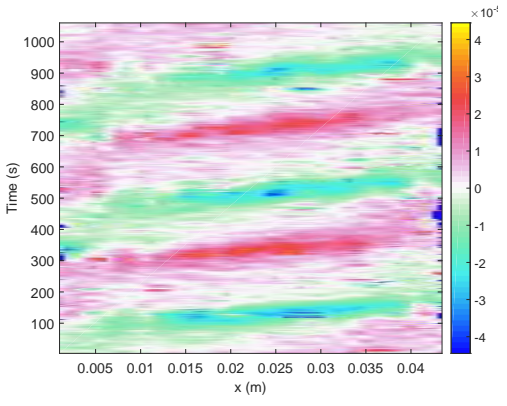

(a) $1 / Y=16.5$

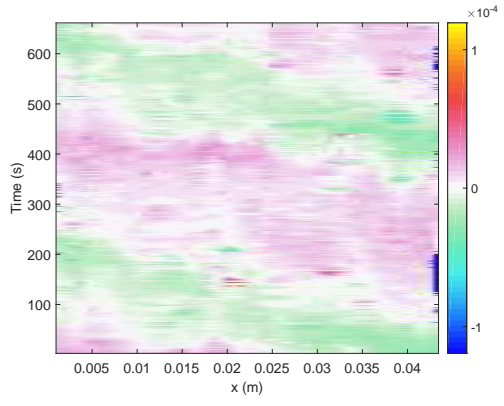

(b) $1 / Y=25$

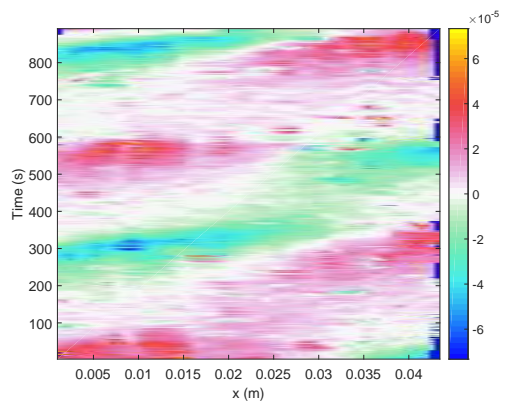

(c) $1 / Y=55$

Figure 11: Space-time diagrams (a)-(c) showing the evolution of the midplane vertical velocity $v_{y}$ as a function of location $x$ obtained in the gel $\left(\tau_{y}=0.03 \mathrm{~Pa}\right)$ for (a) $1 / Y=16.5$, (b) $1 / Y=25$ and (c) $1 / Y=55$.

the vertical velocity $v_{y}$ at the horizontal mid-plane of the cavity for different values of $1 / Y$. The TW periods are similar to the mean period $P$ which corresponds to the time needed for a particle to describe one loop. These phenomena are clearly correlated. During half a period, a particle can describe half a loop, e.g. in the upward direction, then the direction reverses and the particle goes downward and reaches nearly its initial position at one period. This is in agreement with the directions of the velocity vectors in Fig. 10 during one period. However, the TW cover larger distances during one period, meaning that the waves should propagate faster than mean particles. This is confirmed by their phase velocity which is found quasi-constant of the order of $10^{-4} \mathrm{~m} / \mathrm{s}$ as displayed in Fig. 12, for all values of $1 / Y$.

Above the critical threshold $1 / Y_{c}$, a steady state convective regime is obtained. However, the transition between the oscillatory convection and the steady state convection has not been studied in this paper but could be done 


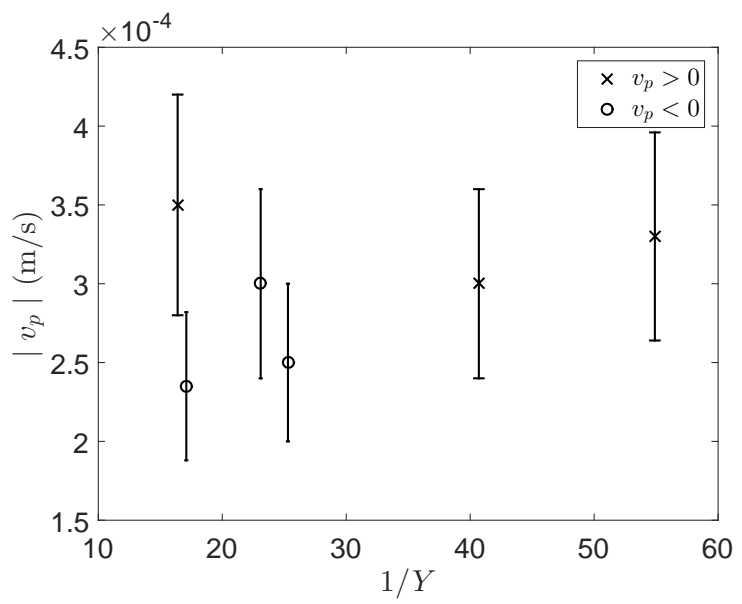

Figure 12: Phase velocity $v_{p}(\mathrm{~m} / \mathrm{s})$ vs. $1 / Y$ of the TW deduced from space-time diagrams

in a future work.

\section{Discussion and conclusion}

The slip boundaries play an important role in the oscillatory convection since such a motion has not been observed with no-slip boundaries. We can assume that the oscillatory motion may be initiated by slippage at walls, especially at horizontal walls where the driven force (or stress) due to buoyancy is maximal. Our results indicate that the gel is sheared in the volume. The accuracy of our results on the walls does not allow us to measure strain at walls correctly. Nevertheless, there is no doubt that sliding has a destabilizing effect, i.e. sliding promotes the occurrence of oscillatory convection. This conclusion is in agreement with theoretical results given for viscoelastic fluids $[2,3,4,1]$. On the other hand, because the bulk gel is sheared, we think that shear-banding, due to inhomogeneity of the stress field [21, 22], remains negligible in our gels.

Oscillations should also be due to elastic effects. While the Carbopol gels behavior is quite different from a Maxwellian fluid, we can only discuss the magnitude orders of the governing parameters which lead to oscillatory convection in viscoelastic fluids. The relaxation time $\lambda$ defined in the Maxwell model corresponds in the linear regime to the ratio between the viscosity $\mu$ of the fluid and the elastic modulus $G^{\prime}$. It is important to underline the 


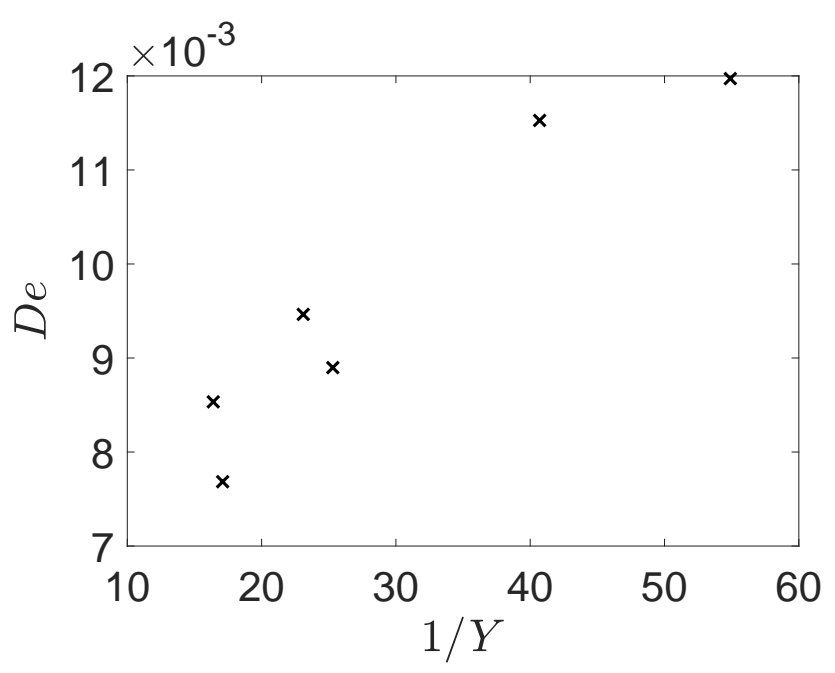

Figure 13: Deborah number vs. $1 / Y$

difficulty of determining a viscosity scale in the unyielded region for stresses close to $\tau_{y}$. Nevertheless, close to the solid-like to liquid-like transition, we consider that the viscosity of Carbopol gels can be scaled by $\mu=\tau_{y} \times T$ as determined by Métivier et al. [17] and based on works of Moller et al. [28] and Dimitriou et al. [29], $T$ is a characteristic time of the material sollicitation. Here, we choose $T=P \approx 500$ s as the characteristic time of the observed phenomenon (period of oscillations). In this case, we get that $\lambda=O(10) \mathrm{s}$ (for $P=500 \mathrm{~s}$ and $\tau_{y}=0.03 \mathrm{~Pa}$, we find $\mu \approx 15 \mathrm{~Pa} . \mathrm{s}$ and $\lambda \approx 20 \mathrm{~s})$. The Deborah number $D e=\lambda \kappa / h^{2}$ is then $D e=O\left(10^{-2}\right)$. Based on this scaling, we evaluate the Deborah number for each yield number, this is displayed in Fig. 13. We observe a slight increase in $D e$ as $1 / Y$ increases. The increase in $D e$ is mainly caused by the increase in relaxation time $\lambda$, highlighting an increase in viscous versus elastic effects. This is related to the increase in buoyancy stresses via the $1 / Y$ increase.

It is worth interesting to scale the viscosity by $\mu=\tau_{f} \times P$ in order to consider the slippage at walls. In this case, we obtain $\lambda \approx 10$ s and $D e \approx 0.005$. Using this viscosity scale, the slip yield stress would have the effect of reducing the Deborah number.

\section{Remark:}

If we evaluate the relaxation time by considering the consistency of the gel, 
i.e. $\lambda=\left(\frac{K}{G^{\prime}}\right)^{-n}$, then we obtain $\lambda \approx 1$ s. The Deborah number is then $D e=O\left(10^{-3}\right)($ for $\lambda=1 \mathrm{~s})$.

On the other hand, we obtain a very large value for the Prandtl number $\operatorname{Pr}=\mu /(\rho \kappa)=O\left(10^{5}\right)$. These values orders for $\operatorname{Pr}$ and $D e=O\left(10^{-2}\right)$ can correspond to the conditions determined theoretically in Maxwell fluids $[1,2,3,4]$ for slip boundaries. Furthermore, the frequencies of oscillations measured in our experiments are also close to the values given by Larson [1] $\omega<10^{-3} \mathrm{~Hz}$.

Similarly to Kolodner [5], we sum up our observations in what follows.

(1) In the Cabopol gel studied in these experiments, the first convective instability is oscillatory when using sliding walls. The oscillations take the form of spatially-localized travelling waves with long oscillation periods $(P \approx 500$ s) and weak amplitudes. Oscillation periods are lower than that obtained by Kolodner's experiments, he obtained periods of the order $10^{4} \mathrm{~s}$.

(2) Typical oscillation frequencies are the order of $\omega=0.001 \mathrm{~Hz}$, leading to a dimensionless frequency of around $\omega^{*}=\omega \frac{d^{2}}{\kappa} \approx 3$. This latter value corresponds to the order of magnitude predicted by earlier theories of viscoelastic convection $\left(\omega^{*} \sim 4-76\right)$.

(3) Similarly to experiments of Kolodner [5], the oscillations in the Carbopol gel are observed at slightly lower values of the Deborah number than the minimum value required by theories. Likewise, our experimental results do not satisfy the criterion given by Sokolov \& Tanner [4] when the number of $\operatorname{Pr}$ tends towards infinity. Indeed, the authors show that oscillatory convection appears if $\frac{G^{\prime \prime}}{G^{\prime} \pi^{2}}>P \frac{\kappa}{d^{2}}$.

(4) In the oscillatory convection regime, runs do not end to steady rolls. Comparison of our results with those of Kolodner shows significant differences in the values of key parameters. These differences are due to the properties of the fluids used, since Kolodner used DNA suspensions (viscoelastic fluids), whereas we experiment elasto-viscoplastic fluids. Furthermore, he assumed no-slip conditions at walls (rigid-rigid boundaries). However, our experimental conditions globally satisfy the theoretical criteria for the onset of oscillatory convection.

The analogy to the oscillation convection in Maxwell fluid tends to demonstrate that elastic effects play the major role in concentrated suspension of 
microgels subject to heat transfer.

Further experiments are needed to characterize in more details the elastic effects and the slippage. The typical structure of the 3D flow field would also be interesting to study. Other gels will be investigated to understand more generally the occurence of this subcritical oscillatory motion. However performing further experimental investigations are made difficult for several reasons. Carbopol's aqueous gels are good model fluids because they are transparent and not thixotropic, but their chemical nature imposes constraints if a yield stress is to be obtained. On the other hand, we are limited in the yield stress range. Indeed, if we want to capture the onset of convection, we have to work with low yield stress values like in the present study. Finally, it would be necessary to decouple the elastic effects from the yield stress effects. But this is hardly possible by experimental means. Indeed, due to the nature of the structure of these microgel suspensions, yield stress and elasticity are linked by fixed laws of scale as shown by Piau [19] for instance. For this aspect, numerical and theoretical works seem more promising. Considering elasto-viscoplastic models such as those proposed by Sarramito [31, 32] for instance, will be the key to describe mechanisms at play. That could answer the question whether the yield stress plays also a role in the occurence of oscillatory convection in Carbopol gels.

\section{Acknowledgments}

The authors are really thankful to Laurent Farge and Julien Boisse for their help in the evaluation of the strain and deviation angle. The LRP is part of the LabEx Tec 21 (grant agreement ANR-11-LABX-0030) and of the PolyNat Carnot Institut (grant agreement ANR-11-CARN-030-01).

\section{References}

[1] R.G. Larson, "Instabities in viscoelastic flows," Rheol. Acta 31, 213 (1992).

[2] T. Green, "Oscillating Convection in an Elasticoviscous Liquid," Phys. Fluids 11, 1410 (1968).

[3] C.M. Vest, and V.S. Arpaci, "Overstability of a viscoelastic fluid layer heated from below," J. Fluid Mech. 36, 613 (1969). 
[4] M. Sokolov, and R.I. Tanner, "Convective Stability of a General Viscoelastic Fluid Heated from Below ," Phys. Fluids 15, 534 (1972).

[5] P. Kolodner, "Oscillatory convection in viscoelastic DNA suspensions," J. Non-Newtonian Fluid Mech. 75, 167 (1998).

[6] H.A. Barnes, "A review of the slip (wall depletion) of polymers solutions, emulsions and particle suspensions in viscosimeters: it causes, character, and cure," J. Non-Newtonian Fluid Mech. 36, 85 (1995).

[7] M. Cloître, and R.T. Bonnecaze, "A review on wall slip in high solid dispersions," Rheol. Acta 56 (2017).

[8] S.P. Meeker, R.T. Bonnecaze and M. Cloître, "Slip and flow of soft particle pastes," Phys. Rev. Lett 92198302 (2004).

[9] J. Seth, M. Cloître and R.T. Bonnecaze , "Influence of short-range forces on wall-slip in microgel pastes," J. Rheol. 521241 (2008).

[10] J. Seth, L. Mohan, C. Locatelli-Champagne, M. Cloître and R.T. Bonnecaze, "A micromechanical model to predict the flow of soft particle glasses," Nat. Mater. 10838 (2011).

[11] A.L Vayssade, C. Lee, E. Terriac, F. Monti, M. Cloître and P. Tabeling, "Dynamical role of slip heterogeneities in confined flows," Phys. Rev. E 89052309 (2014).

[12] J. Zhang, D. Vola and I.A. Frigaard, "Yield stress effects on RayleighBénard convection," J. Fluid Mech. 566, 389 (2006).

[13] A. Vikhansky "Thermal convection of a viscoplastic liquid with high Rayleigh and Bingham numbers," Phys. Fluids 21103103 (2009).

[14] M. Darbouli, C. Métivier, J.-M. Piau, A. Magnin, and A. Abdelali "Rayleigh-Bénard convection for viscoplastic fluids," Phys. Fluids 25 023101 (2013).

[15] Z. Kebiche, C. Castelain, and T. Burgelea, "Experimental investigation of the Rayleigh-Bénard convection in a yield stress fluids," J. NonNewtonian Fluid Mech. 203, 9 (2014). 
[16] C. Li, A. Magnin and C. Métivier "Natural convection in shear-thinning yield stress fluids in a a square enclosure," Aiche J. 62(4) 1347 (2016).

[17] C. Métivier, C. Li and A. Magnin "Origin of the onset of RayleighBénard convection in a concentrated suspension of microgels with a yield stress behavior," Phys. Fluids 25023101 (2017).

[18] C. Métivier, Y. Rharbi, A. Magnin and A. Bou Abboud, "Stick-slip control of the Carbopol gels on transparent smooth walls," Soft Matter, 8, 7365 (2012).

[19] J.-M. Piau, "Carbopol gels: Elastoviscoplastic and slippery glasses made of individual swollen sponges: Meso- and macroscopic properties, constitutive equations and scaling laws," J. Non-Newtonian Fluid Mech. 144, 1 (2007).

[20] F. Ahonguio, L. Jossic, A. Magnin, "Influence of surface properties on the flow of a yield stress fluid around spheres," J. Non-Newtonian Fluid Mech. 206 (2014).

[21] T. Divoux, D. Tamarii, C. Barentin, and S. Manneville, "Transient shear banding in a simple yield stress fluid," Phys. Rev. L 104, 208301 (2010).

[22] G. Ovarlez , S. Cohen-Addad, K. Krishan, J. Goyon, and P. Coussot, "On the existence of a simple yield stress fluid behavior," J.NonNewtonian Fluid Mech. 193 (2013).

[23] M. Drinkgreve, M. Fazilati, M.M. Denn, and D. Bonn, "Carbopol: From a simple to a thixotropix yield stress fluid," J. Rheol. 62(2) (2018).

[24] R.J. Schmidt, and S.W. Milverton, "On the instability of a fluid when heated from below," Proc. Roy. Soc. (London)A. 152, (1935).

[25] R. Baude and M. Desecures, "TRACK software". http:///www.aprexsolutions.com.

[26] F. Brochard, V. Rohde, T. Lunt, G. Suarez Lopez, A. Shalpegin and R. Neu, Intrinsic dust transport in ASDEX Upgrade studied by fast imaging, Nuclear Materials and Energy 18, 268 (2019). 
[27] Bayesian Probability Theory, Applications in the Physical Sciences, W. von der Linden, V. Dose, U. von Toussaint, Cambridge University Press (2014).

[28] Moller P., Fall A., and Bonn D., Origin of apparent viscosity in yield stress fluids below yielding, Europhys. Lett. 87, 38004 (2009).

[29] C.J. Dimitriou, R.H. Ewoldt, G.H. McKinley, "Describing and prescribing the constitutive response of yield stress fluids using large amplitude oscillatory shear stress (LAOStress)," J. Rheol. 571 (2013).

[30] P. Coussot, "Slow flows of yield stress fluids: yielding liquids or flowing solids?," Rheol. Acta 57 (2018).

[31] P. Saramito, "A new constitutive equation for elastoviscoplastic fluid flows," J. Non-Newtonian Fluid Mech. 145, 1 (2007).

[32] P. Saramito, "A new elastoviscoplastic model based on the HerschelBulkley viscoplastic model," J. Non-Newtonian Fluid Mech. 158, 1 (2009). 